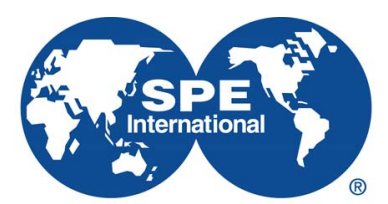

Society of Petroleum Engineers

\title{
SPE-196824-MS
}

\section{Implementation of Different Types Flowmeters for Gas Lift Metering on Orenburgskoe Field}

Konstantin Rymarenko, Siant; Marat Nukhaev, Siberian Federal University; Sergey Grishenko, Nikita Chernysh, and Sergey Titov, Siant; Alexander Zaycev, Novosibirsk State University; Nikita Dadakin, Siberian Federal University; Vladimir Ulyanov, Gazpromneft

Copyright 2019, Society of Petroleum Engineers

This paper was prepared for presentation at the SPE Russian Petroleum Technology Conference held in Moscow, Russia, 22 - 24 October 2019.

This paper was selected for presentation by an SPE program committee following review of information contained in an abstract submitted by the author(s). Contents of the paper have not been reviewed by the Society of Petroleum Engineers and are subject to correction by the author(s). The material does not necessarily reflect any position of the Society of Petroleum Engineers, its officers, or members. Electronic reproduction, distribution, or storage of any part of this paper without the written consent of the Society of Petroleum Engineers is prohibited. Permission to reproduce in print is restricted to an abstract of not more than 300 words; illustrations may not be copied. The abstract must contain conspicuous acknowledgment of SPE copyright.

\section{Abstract}

Gas or liquid flow control is one of the most important technical tasks in the oil and gas industry. The choice of a particular device most often occurs on the basis of the established practice of application in a particular company. This paper presents the experience of Gazpromneft-Orenburg in the use of overhead and stationary flow meters of various types, including ultrasonic and restriction devices for measuring gaslift gas in the Orenburg oil and gas condensate field.

\section{Gas lift wells operation at the Orenburg oil and gas condensate field}

At present, Gazpromneft-Orenburg uses gas-lift operation at most production wells in the Eastern part of the Orenburg oil and gas condensate field (Galyautdinov, 2017). This method involves the injection of gas through the annulus to the bottom of a well, where it enters through a working gas-lift valve into the string of lift pipes and mixes with the well fluid. The density of the fluid in the tubing is reduced and the well can operate without the involvement of other methods of artificial lift. The gas-lift method of operation has become widespread throughout the world because of its clear advantages over other methods of artificial lift, such as:

- Ability to select large volumes of fluid with virtually all diameters of production casing and speeded up sampling of high water-cut wells

- Operation of wells with a high gas factor, i.e. utilization of reservoir gas energy

- Low influence of the wellbore profile on the gas lift performance, which is especially important for directional wells, i.e. for the conditions of offshore fields and the North development areas and Siberia

- Lack of influence of high pressures and temperature of well production, as well as the presence of mechanical impurities (sand) in it on the work of wells

- Flexibility and comparative ease of wells flow rate controlling 
- Ease of maintenance and repair of gas-lift wells and a long turnaround time for their work when using modern equipment

- The ability to use simultaneous separate operation, effective corrosion control, salt deposits and paraffin

- Ability and ease of field well testing.

In addition to the presence of hydrogen sulfide (up to $6 \% \mathrm{H} 2 \mathrm{~S}$ ) the features of gas lift operation at the Orenburg oil and gas condensate field, include difficulties associated with the presence of a liquid phase in gas lift gas caused by the following factors (Trubavin, 2017):

- A part-free gas lift is used on part of the wells. With this method of operation, the gas from the gas production well does not undergo dehumidification and is supplied as gas-lift gas to the production wells together with drops of liquid hydrocarbons and water.

- When daily temperature fluctuations reach $40^{\circ} \mathrm{C}$, the effect of a sharply-continental climate leads to condensation

- When conducting well surveys, the situation is aggravated by a change in the operating modes of the well and, accordingly, a change in the operating modes of the gas-lift gas supply line. When changing the gas-lift gas flow rate, the pressure wave disrupts condensate droplets from the pipe walls, which leads to an increase in the proportion of liquid in the flow.

In these conditions, one of the key tasks is the selection of the optimal measurement technology for gas-lift gas consumption. The importance of this task is related to the fact that in order to optimize the operation of gas-lift wells, it is necessary to construct the characteristic of the wells operation in the form of dependence of the liquid flow rate on the gas-lift gas flow rate (Shushakov, 2015). After obtaining this dependence, the gas-lift well is adjusted for optimal operation by maintaining the gas-lift gas flow rate in the area of rational operation of the lift (Figure 1).

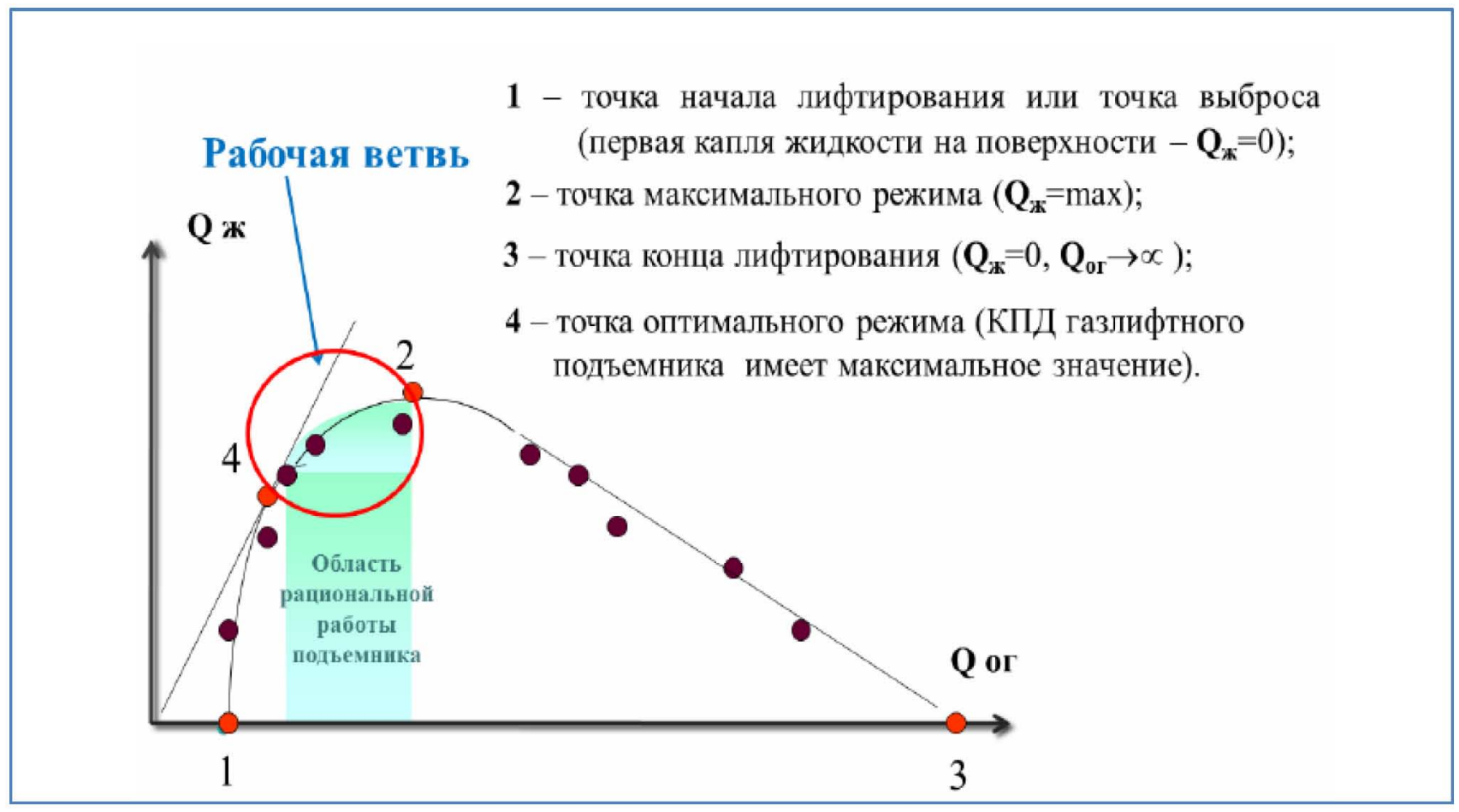

Figure 1-Characteristics of the gas-lift well 


\section{Overview of various types of flowmeters}

In the oil industry, single-phase flow meters are traditionally called meters. There are a huge number of different types of single-phase flow meters, differing not only in size and metering characteristics, but also in various physical measurement principles. These types of flow meters include: mechanical, Coriolis, vortex, turbine, ultrasonic, flow meters of variable pressure drop (diaphragm, Venturi), thermal, and many others.

Turbine flowmeters are high-speed flowmeters in which the kinetic energy of the measured flow is used to create torque on the measuring impeller. The principle of operation of the wing-tachometric flow meters designed to measure flow is as follows. A balanced light impeller rotating in bearings with low friction is placed in the measured flow. The impeller under the pressure of a moving stream rotates. In stationary mode, the speed of its rotation is proportional to the flow rate. Structurally, the impeller can be axial or tangential (Figure 2). Measurement of the impeller speed can be done in various ways: electric, radioactive, photoelectric, etc. The resulting pulsating electrical signal, the number of pulsations of which per time unit is proportional to the number of the impeller rotations, after amplification is fed to a frequency meter, the measuring signal from which is fed to a recording device. The number of pulses registered (counted) by the system per time unit, characterizes the consumption of the measured substance.
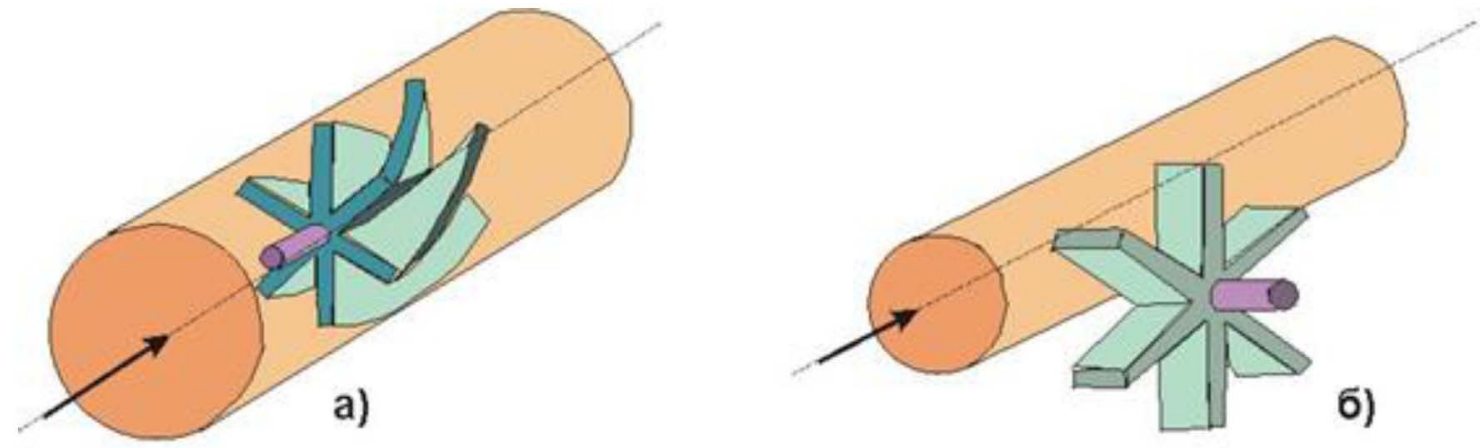

\section{а-аксиальная; б-тангенциальная}

Figure 2-Turbine flow meter schematic diagram

The main operational factors that significantly affect the flow measurement accuracy of turbine flow meters are:

- change in viscosity of the measured medium

- supports wear-out

- flow spin caused by the influence of local resistance.

As a result, these devices are not very suitable for measuring the flow of polluted or abrasive media, as well as liquids, which greatly change their viscosity. There is also a high risk of hydrate formation for the conditions of the Orenburg oil and gas condensate field.

The principle of operation of Coriolis flowmeters is based on the fact that an additional movement is reported to the flow of the measured substance in order to create inertial effects in the flow, which are used to assess the mass flow rate. In this regard, these flow meters are also called inertial. Depending on what kind of additional movement is communicated to the flow (by means of a rotating or oscillating link), either a Coriolis force, or a gyroscopic, or inertial moment occurs on the sensitive element of the device. Coriolis flow meters have the following advantages over other types of flow meters: straight pipe sections before and after the flow meter are not required, independence from fluid viscosity. The disadvantages include 
the relatively low accuracy of gyroscopic flow meters, high sensitivity to heterogeneity (multiphase) flow, incompatibility with the presence of solid particles (hydrates) and difficulty in operation.

A vortex flowmeter is a type of flowmeter whose principle of action is based on measuring the frequency of oscillations that occur in a stream in the process of vortex formation. In vortex flowmeters, a flow body is installed to create a vortex motion in the path of a moving stream of liquid or gas (Figure 3 ). The system of vortices formed behind it is called the Karman vortex path. In the first approximation, the frequency of the vortices is directly proportional to the flow velocity, inversely proportional to the width of the flow body and depends on the dimensionless criterion (Strouhal number). The advantage of vortex flowmeters is the invariance of the method with respect to electrical properties and the state of aggregation of the moving medium. The disadvantage is low accuracy in an inhomogeneous medium and in conditions of varying composition, as well as channel geometry with sharp protruding elements (moisture accumulates on these elements and stagnant zones behind them, and therefore there is a high risk of hydrate formation).

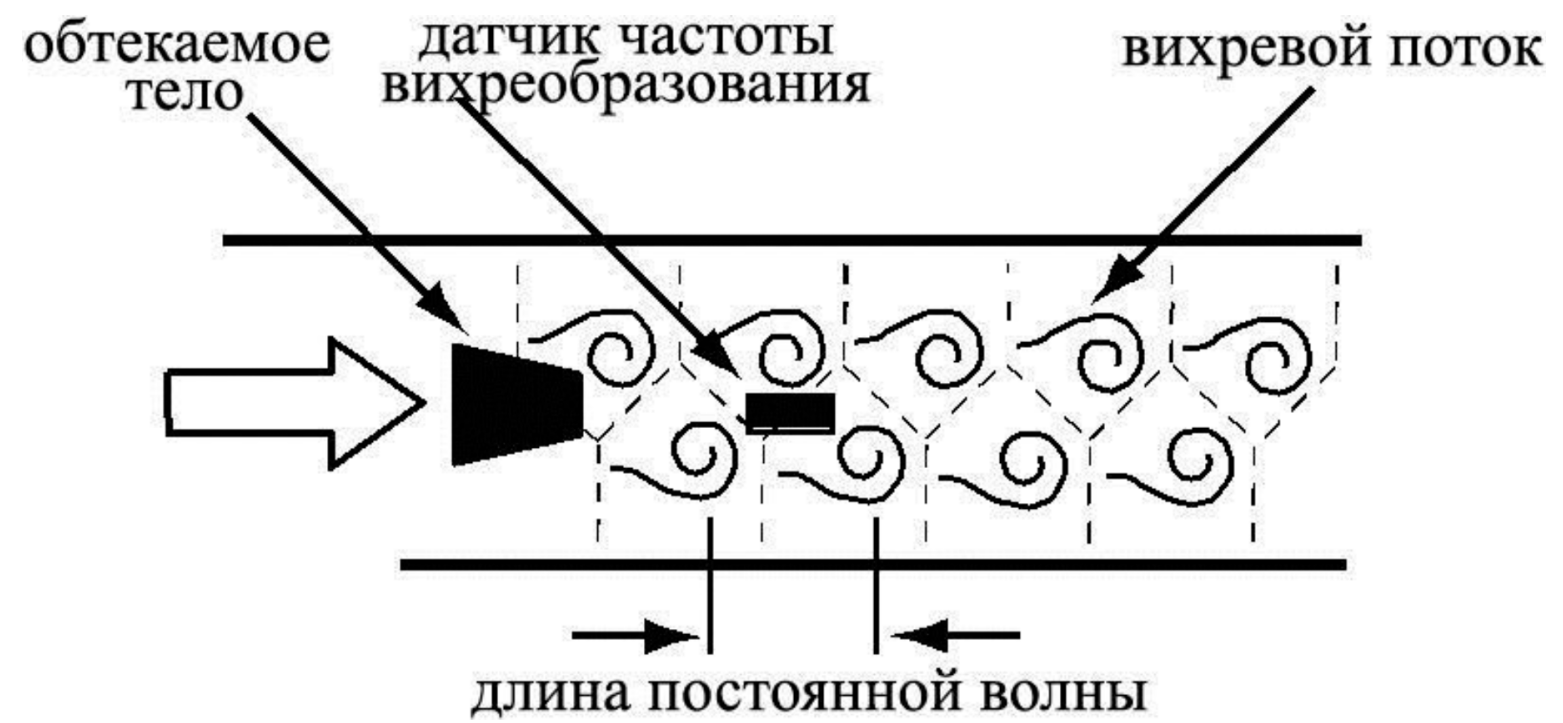

Figure 3-Vortex flow meter schematic diagram

In thermal flow meters, the flow velocity is measured either by cooling a heated body placed in a flow or by transferring thermal energy between two points along the flow. Depending on this, heat flow meters can be divided into thermoanemometers, the operation principle of which is based on the entrainment of heat from the heated body and measuring the electrical parameters of the latter and calorimetric flowmeters, the principle of action of which is based on the heat transfer from the heater to the appropriate meter. The intensity of the transfer of heat energy by the studied flow is a measure of the flow velocity. In such devices, thermal energy plays the role of an intermediate measurement parameter. The main disadvantage of heat flow meters is a significant impact on their readings of temperature, pressure and various thermal parameters of the flow. In the conditions of the Orenburg oil and gas condensate field, daily temperature fluctuations are quite noticeable, as well as temperature drops in the flow when choking the gas-lift gas line.

Among ultrasonic flowmeters most common for measurements in the oil and gas industry are timepulse flowmeters. Such flowmeters measure the difference in the time of transmission of an ultrasonic wave in the direction and against the direction of flow of a liquid or gas. The principle of operation of ultrasonic flow meters is based on measuring the difference in signal transmission time. In this case, two ultrasonic sensors, located diagonally opposite each other, function alternately as a transmitter and receiver. Thus, the acoustic signal, alternately generated by both sensors, is accelerated when directed downstream and slows down when directed upstream. In this way, it is possible to find the average flow rate along the signal path, on the basis of which the volume flow can then be calculated under operating conditions (pressure and 
temperature for a given medium composition). To compensate for the distortion of the flow profile, several acoustic channels are used. At the same time, this type of flow meter works well only for a pure stream or a stream with an insignificant content of suspended particles.

Variable pressure flow meters (which include flow meters with restriction devices) provide significantly less resistance to flow than mechanical and other types of flow meters. Flow meters of this type use the laws of pressure change in moving liquids and gases under varying conditions (for example, different crosssectional area for a restriction device using the Bernoulli law or a different direction of the velocity vector of entry into the differential pressure sensor for the Pitot tube). The most accurate flowmeter with a restriction device is a flowmeter with a Venturi due to the geometry of the restriction (Aksenov, 2013). It is designed to prevent significant non-uniformity of the flow profile (which is important for measuring pressure by sensors that interact with the medium only along the section contour) and to reduce turbulence (which also affects the measurement accuracy).

\section{The need to create a new flow meter for a heterogeneous type of fluid}

To improve the efficiency of operation of wells in the Orenburg oil and gas condensate field, the Gazlift automated process control system (Ulyanov, 2018) is used. This complex is designed to control the process of gas-lift oil production by monitoring and remote control, including:

- Collection and processing of information from primary transducers

- Control of actuators to ensure optimal well performance

- Operational change of well operation mode

- Accumulation of the obtained information and monitoring of the state of oil wells (Figure 4).

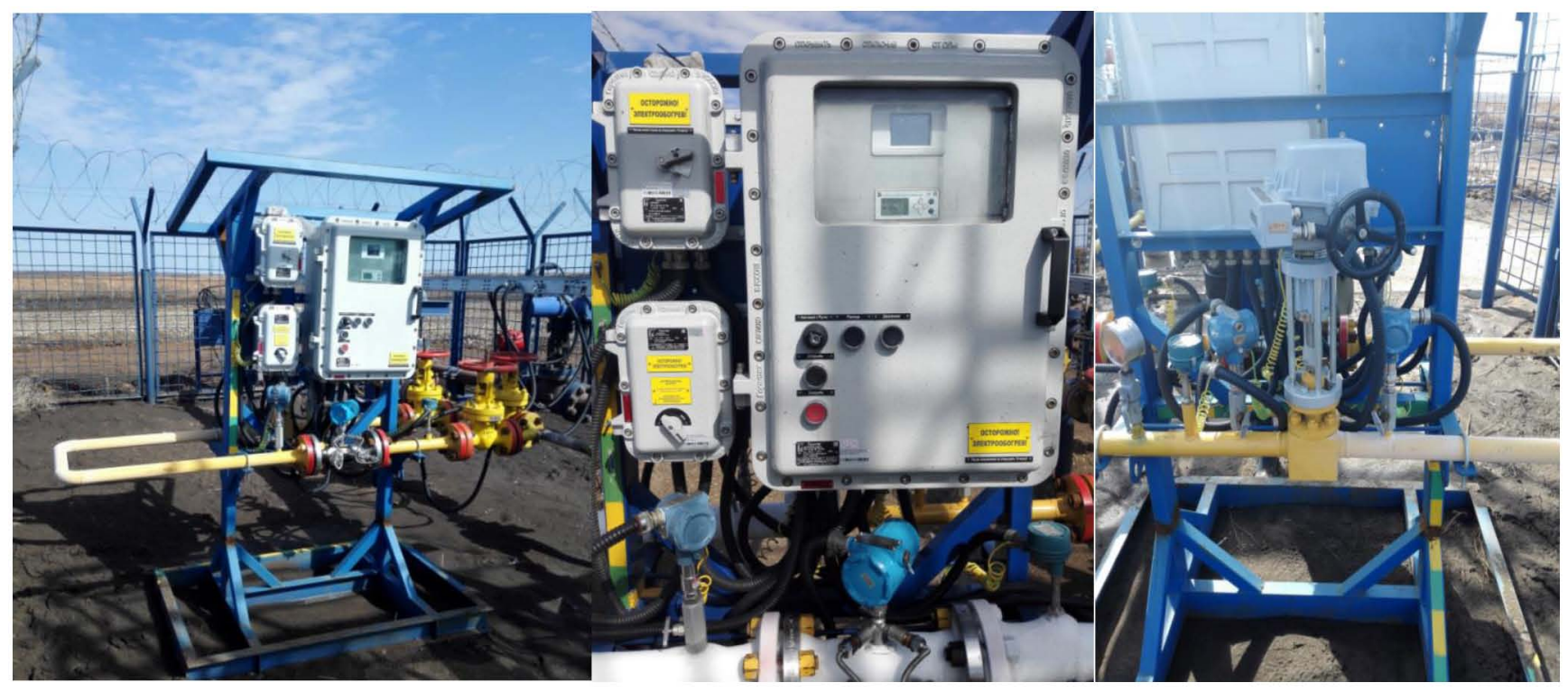

Figure 4-Gazlift automated process control system

The introduction of the Gazlift automated process control system allows Gazpromneft-Orenburg not only to optimize the operation of the gas-lift wells, but also to optimize work in general at the field, including due to:

- freeing up time for engineers to analyze and make decisions by reducing the workload for routine operations 
- optimization of operators work due to remote control of technological objects and wells

- reducing the risks of occupational safety and health of personnel in conditions of high content of hydrogen sulfide.

In the process of industrial implementation of the Gazlift automated process control system the following problems were found:

- formation of hydrates in the gas supply lines due to the increased moisture content in the gas-lift gas

- significant fluctuations in the ultrasonic flow meters readings.

The problem of hydrate precipitation was solved with the help of several solutions: injection of methanol into gas-lift gas, use of a heating cable. A reactive algorithm to destroy the formation of gas-hydrates was also used, which is as follows (Figure 5):

- the Gazlift automated process control system monitors specific signatures of hydrate formation (according to the indications of the ultrasonic flow meter, the behavior of the readings by the pressure and temperature sensor before and after the valve)

- in the case of the risk of hydrates, reactive algorithm to destroy the formation of gas-hydrates is activated

- $100 \%$ of the valve opens at its maximum rapidity in order to purge the gas-lift gas supply line for a certain time. The valve is in this position for a certain time, after which the valve closes as quickly as possible

- the algorithm is repeated several times, and then the well is transferred to normal operation.

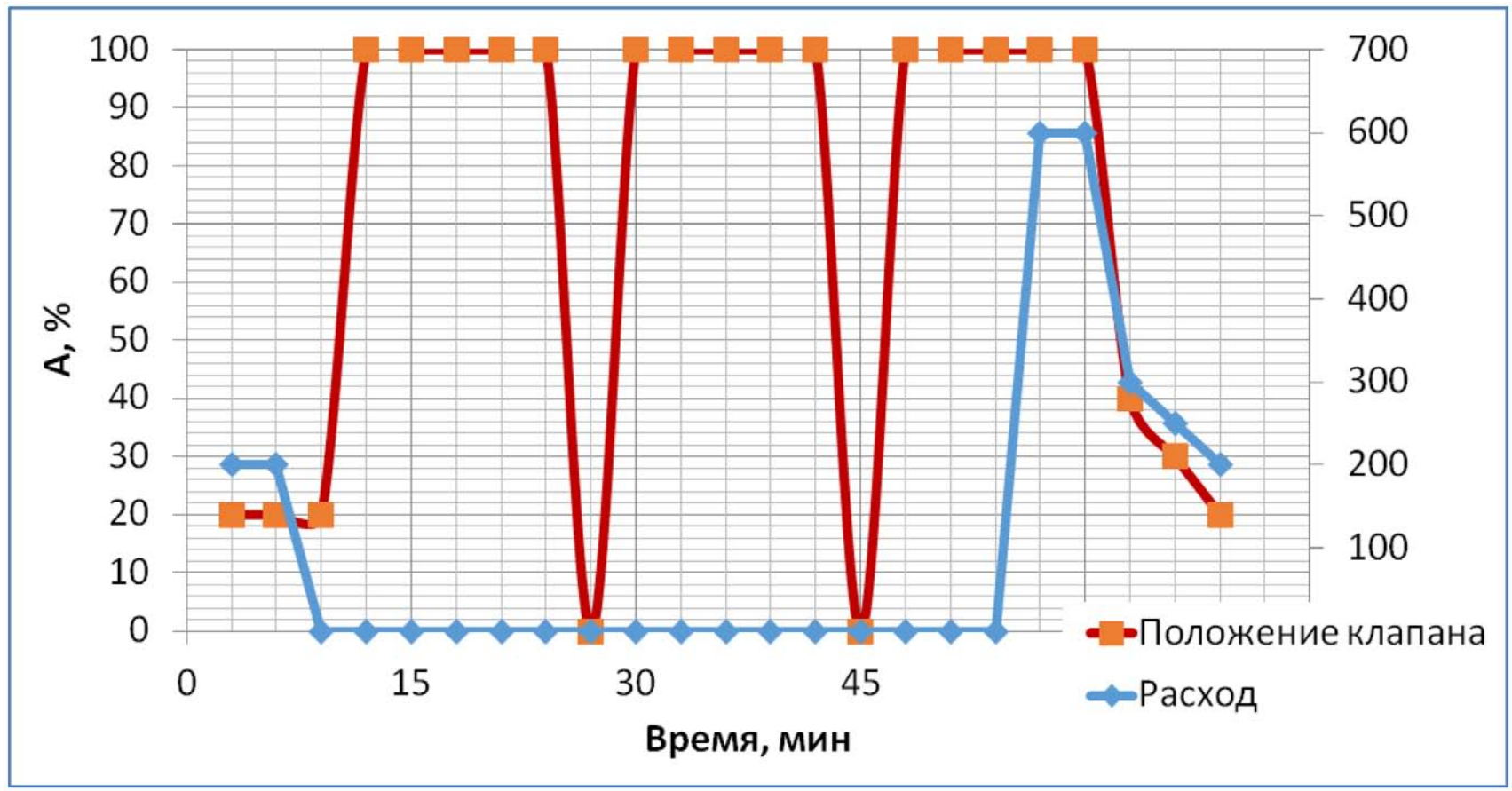

Figure 5-A reactive algorithm to destroy the formation of gas-hydrates by purging the gas-lift supply line

The problem of fluctuations in the ultrasonic flow meters readings has a serious impact not only on the measurement of the well and well testing, but also on the operation of the automated process control system. For example, Figure 6 shows the operation of the valve of the automated process control system at various settings for gas lift gas flow. The green line is the reading of an ultrasonic stationary flow meter. It can be seen that the readings of the ultrasonic stationary flow meter deviate from the setting value by $+/-40 \%$. 
The needle of the valve responds accordingly (\% of area openness) while constantly in motion in order to reach the preset set point of gas-lift gas consumption. This leads to a decrease in valve operating time and unstable system operation. The reason for such fluctuations in the indications of the ultrasonic flow meter is the presence of a liquid phase in gas-lift gas, as well as changes in the system operation mode (when switching from one set point to another), which cause condensate droplets to fall off the pipe walls, which leads to an increase in the proportion of liquid in the flow.

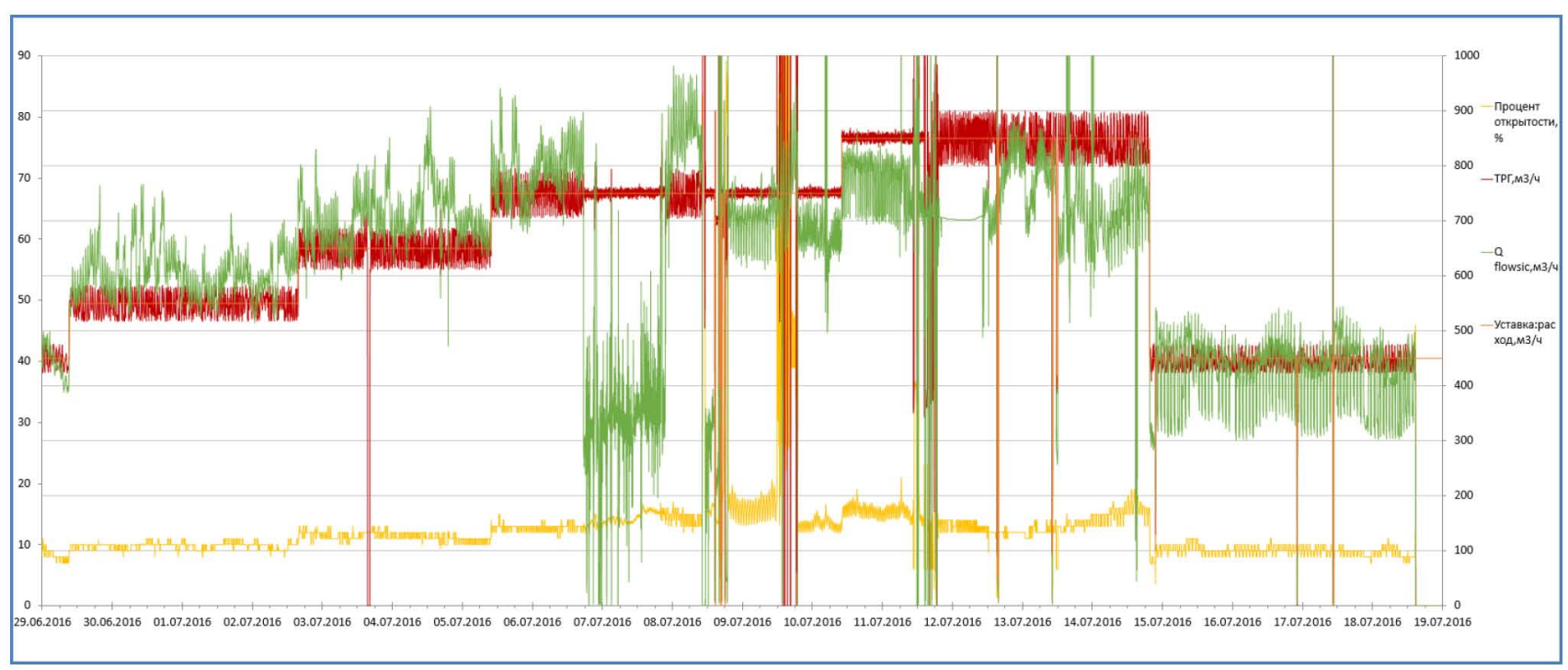

Figure 6-Ultrasonic flow meter reading

Thus, for proper well testing and optimal output to the gas-lift well mode in the conditions of the Orenburg oil and gas condensate field, a flow meter is required for a heterogeneous type of fluid.

\section{Simulation of flow meter operation based on a restriction device}

Gazpromneft-Orenburg together with SIANT Research and Production Company decided to create a flow meter for operation for a substantially heterogeneous environment based on a restriction device. The following restriction devices were considered: the diaphragm/nozzle and a Venturi tube.

To select and justify the type of a restriction device, numerical experiments were performed using CDF simulation. Figure 7 shows the pressure distribution during gas flow through the Venturi and nozzle. For an inlet pressure of 100 bars and a gas flow rate of $5000 \mathrm{~m} 3 / \mathrm{h}$, the pressure loss for the Venturi tube was 5.8 $\mathrm{kPa}$, and the flow rate error was $\sim 0.5 \%$. For a restriction device in the form of a nozzle for an input pressure of 100 bars and a gas flow rate of $5000 \mathrm{~m} 3 / \mathrm{h}$, the pressure loss was $35 \mathrm{kPa}$, and the flow rate error was $\sim 3 \%$. 

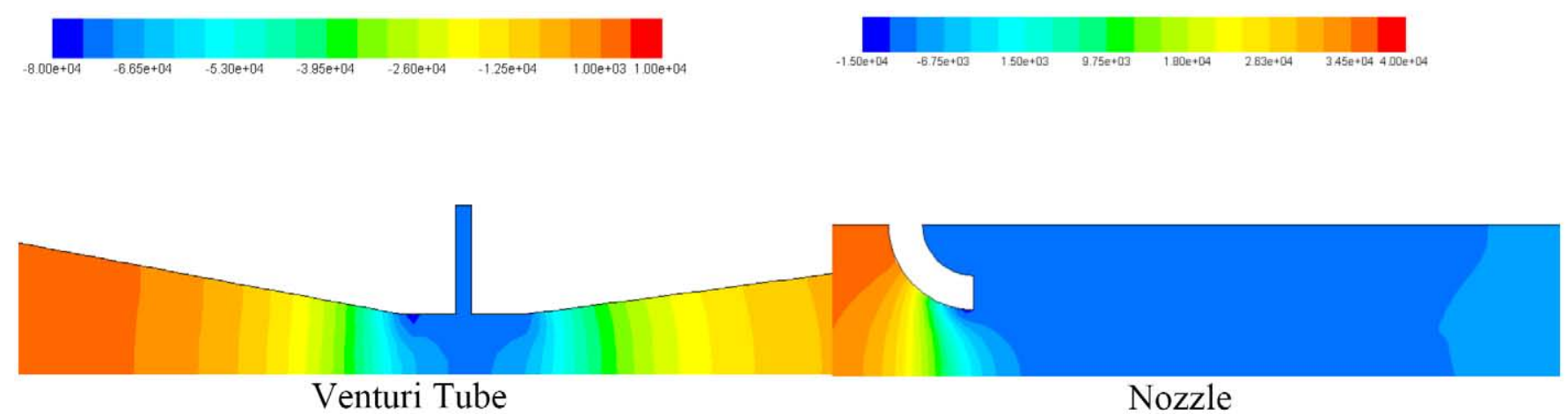

Figure 7-Pressure distribution during gas flowing through a Venturi tube and nozzle

The presence of stagnant zones or the presence of large-scale vortices in the channel can create favorable conditions for gas hydrate deposits. Figure 8 presents a picture of the flow by a vector field coloured according to the absolute amount of the gas velocity. For the outflow from the nozzle, large stagnant zones are typical, and for the Venturi tube they are virtually invisible.
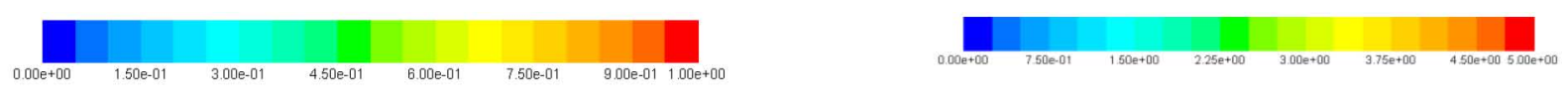

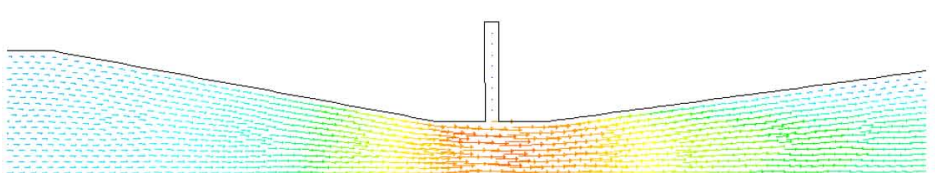

Venturi Tube

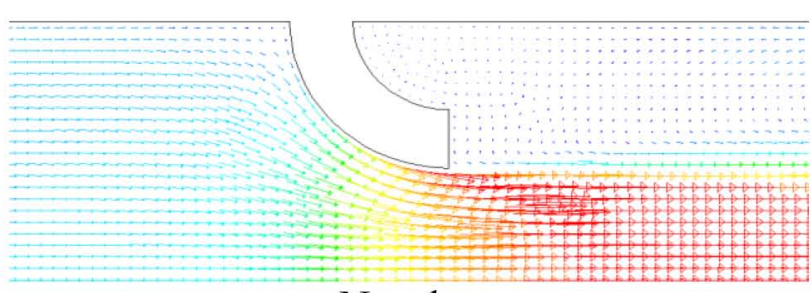

Nozzle

Figure 8-The distribution of velocity and direction of gas flow for a Venturi tube and nozzle.

To study the potential formation of gas hydrates deposits, it is important to know the areas of accumulation of water that may present in the flow in the form of small droplets. Figure 9 shows the results of numerical simulation of possible trajectories of movement of water droplets with a diameter of $0.5 \mathrm{~mm}$. A sufficiently large amount of water will be deposited on the front wall of the nozzle, at the same time they will pass through a Venturi tube without contact with the walls.

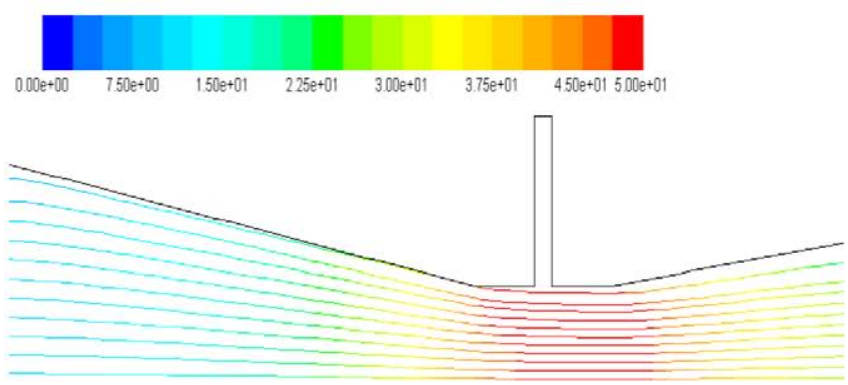

Venturi Tube
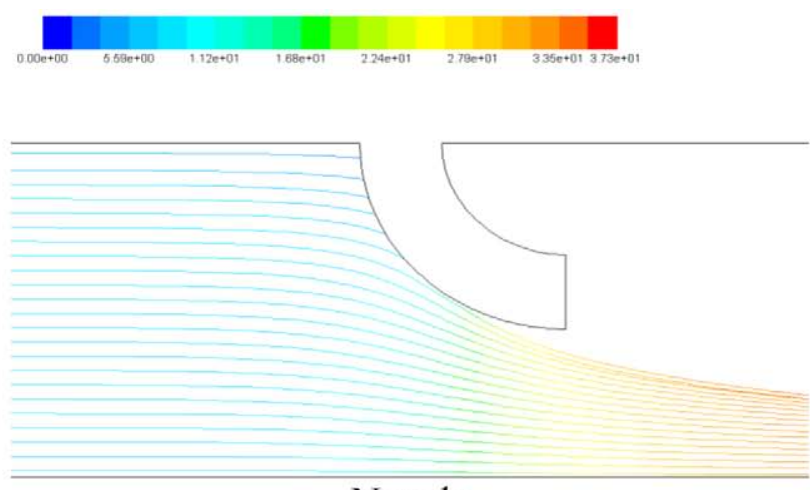

Nozzle

Figure 9-Derictions and velocity for gas flowing through a Venturi tube and nozzle. 
After the results of the CDF simulation, it was decided to choose a flowmeter on the basis of a Venturi tube due to the following reasons (despite the fact that it is the most difficult device for manufacturing from all types of restriction devices):

- No significant pressure loss in the flow

- No stagnant zones. Reduced risk of hydrate formation

- Low sensitivity to moisture in the measured gas medium.

The last two points are especially important for the operating conditions of the Orenburg oil and gas condensate field.

\section{Testing flow meter on wet stand}

Liquid and gas flow meter based on a restriction device such as Venturi flow meter "FlowMaster" is a classic flowmeter of this principle of operation with some modifications (Figure 10). Its mechanical part is made in compliance with the principle of modularity and allows the use of a device with a third-part computer. This can be convenient when using the flow meter as part of any automated complex. At the same time, parts that are not intended for separate operation constitute a solid block, which increases the reliability of the device and reduces its price. The block consists of a gas line with flange connectors, narrowing with all necessary channels or capillaries (integrating, measuring, technological), taps for connecting additional sensors (overpressure and medium temperature).

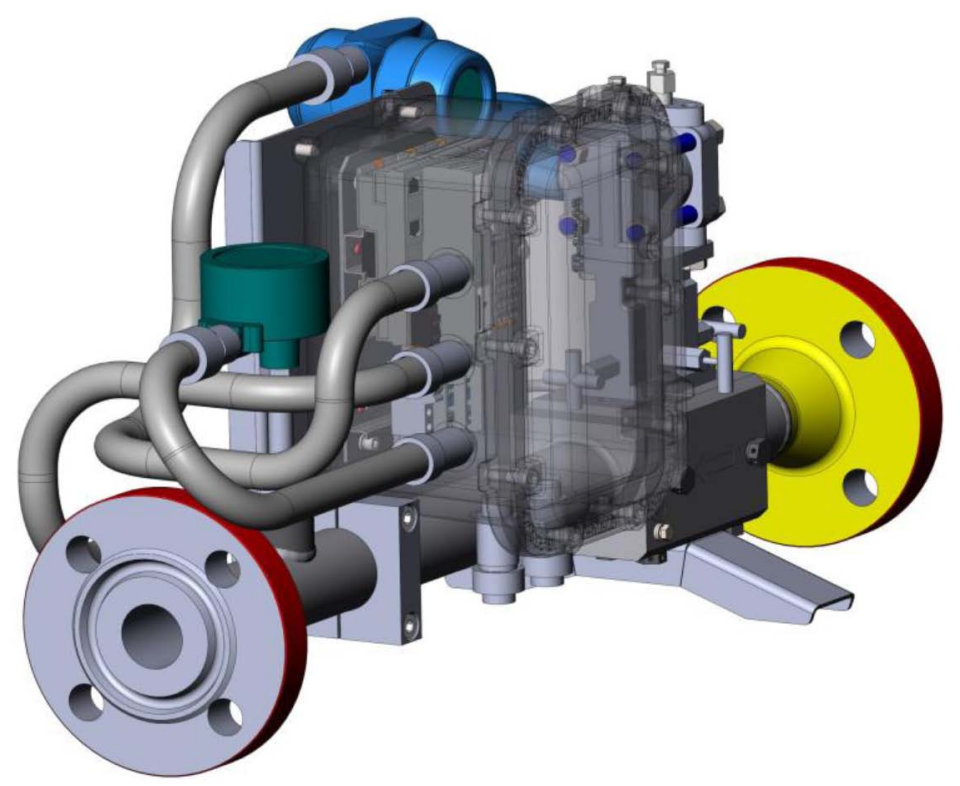

Figure 10-3D diagram of a Venturi tube prototype

For carrying out experiments on a wet stand, two prototypes of a Venturi tube with diameters of critical sections 19 and $22 \mathrm{~mm}$ were manufactured. In 2018, a prototype of a flow meter was tested at the Institute of Theoretical and Applied Mechanics of Siberian Branch of the Russian Academy of Sciences on a wet stand. The device was connected to an experimental test bench, where the gas (air) flow was measured using throttle washers with a diameter of 2, 3 and $5 \mathrm{~mm}$. The wiring diagram is shown in Figure 11. The diameter of the wide part of the channel is $\mathrm{D}=45 \mathrm{~mm}$, the diameter of the critic is $\mathrm{d}=19 \mathrm{~mm}$ and $22 \mathrm{~mm}$. Figure 12 shows a photograph of the experimental setup. Figure 13 presents a comparison of the theoretical gas consumption calculated by the adiabatic formula and gas consumption measured by the FlowMaster. 


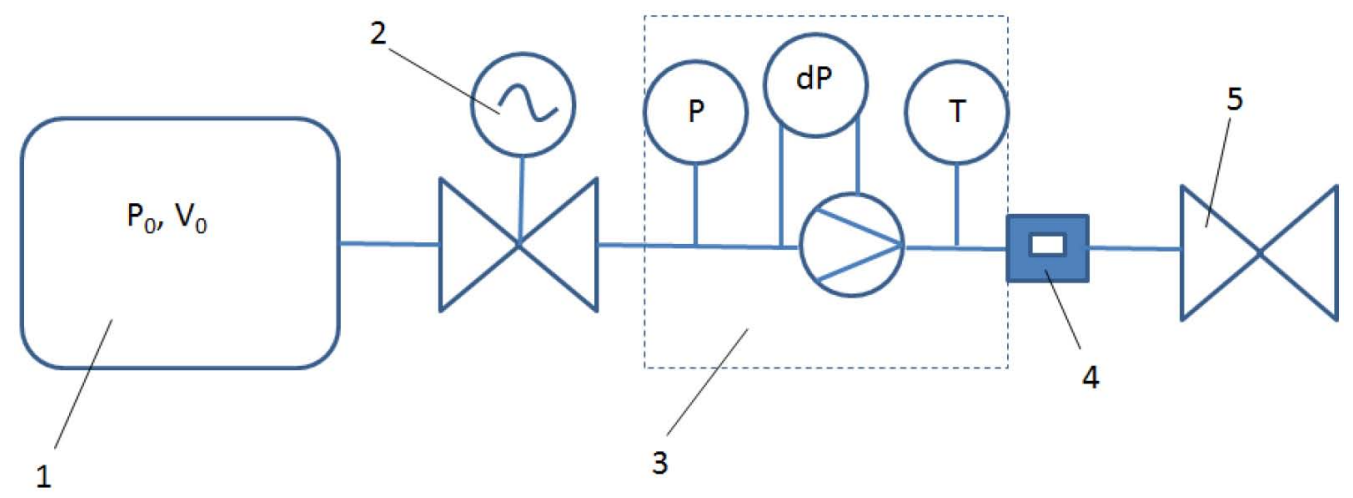

Figure 11-Diagram of the experimental stand 1 - high-pressure air cylinder with a volume of 100 . The initial pressure and volume of the cylinder are selected for the pressure and flow ranges of interest, and should provide an experiment for about a minute $\mathbf{2}$ - adjustable valve with a large bore $\mathbf{3}$ - flow meter and a set of measuring devices 4 - throttle (consumable) washer, replaced in accordance with the required flow rate in each specific experiment 5 - shut-off output valve of a large bore, or high-speed valve.

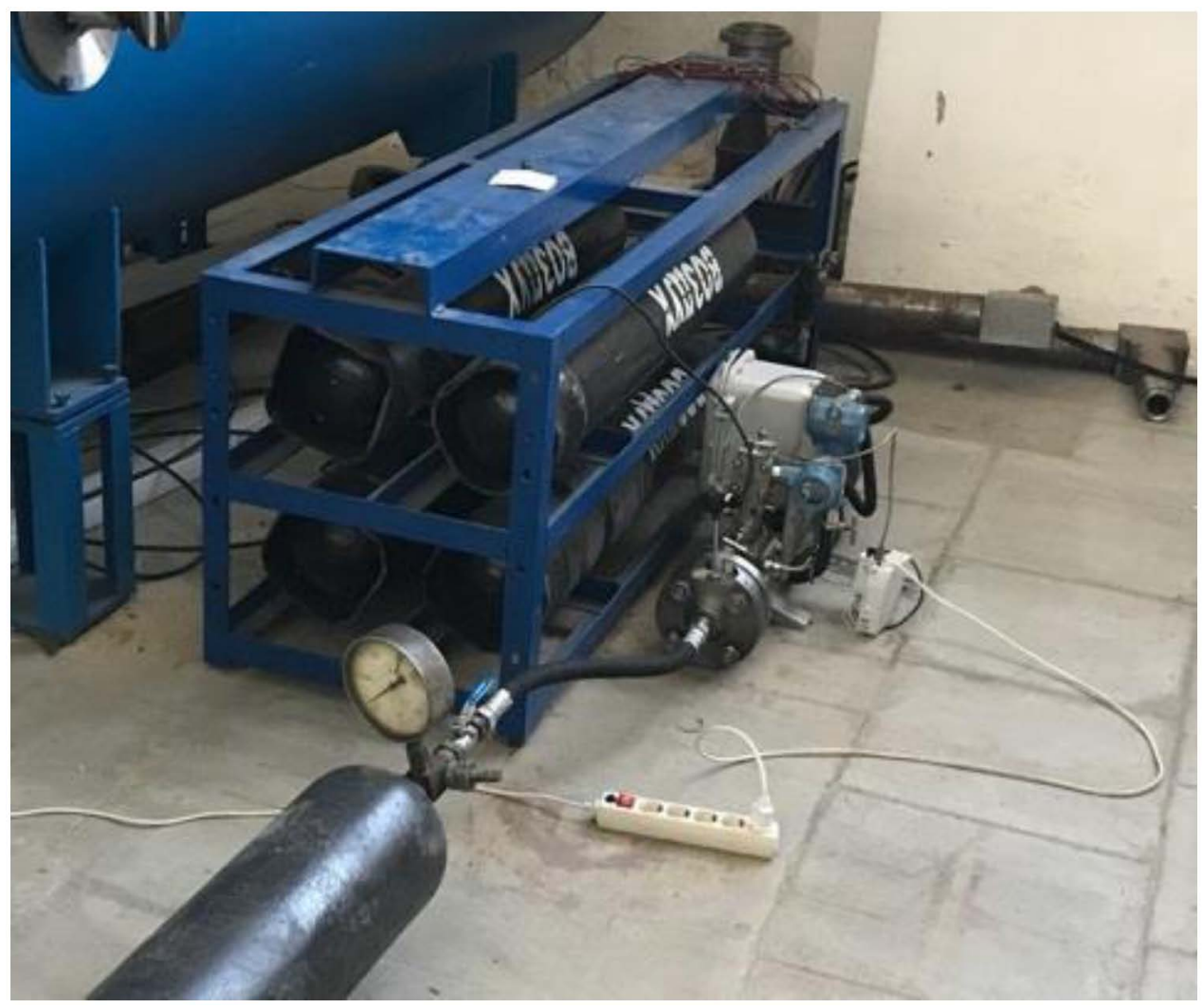

Figure 12-Photograph of the experimental stand. 


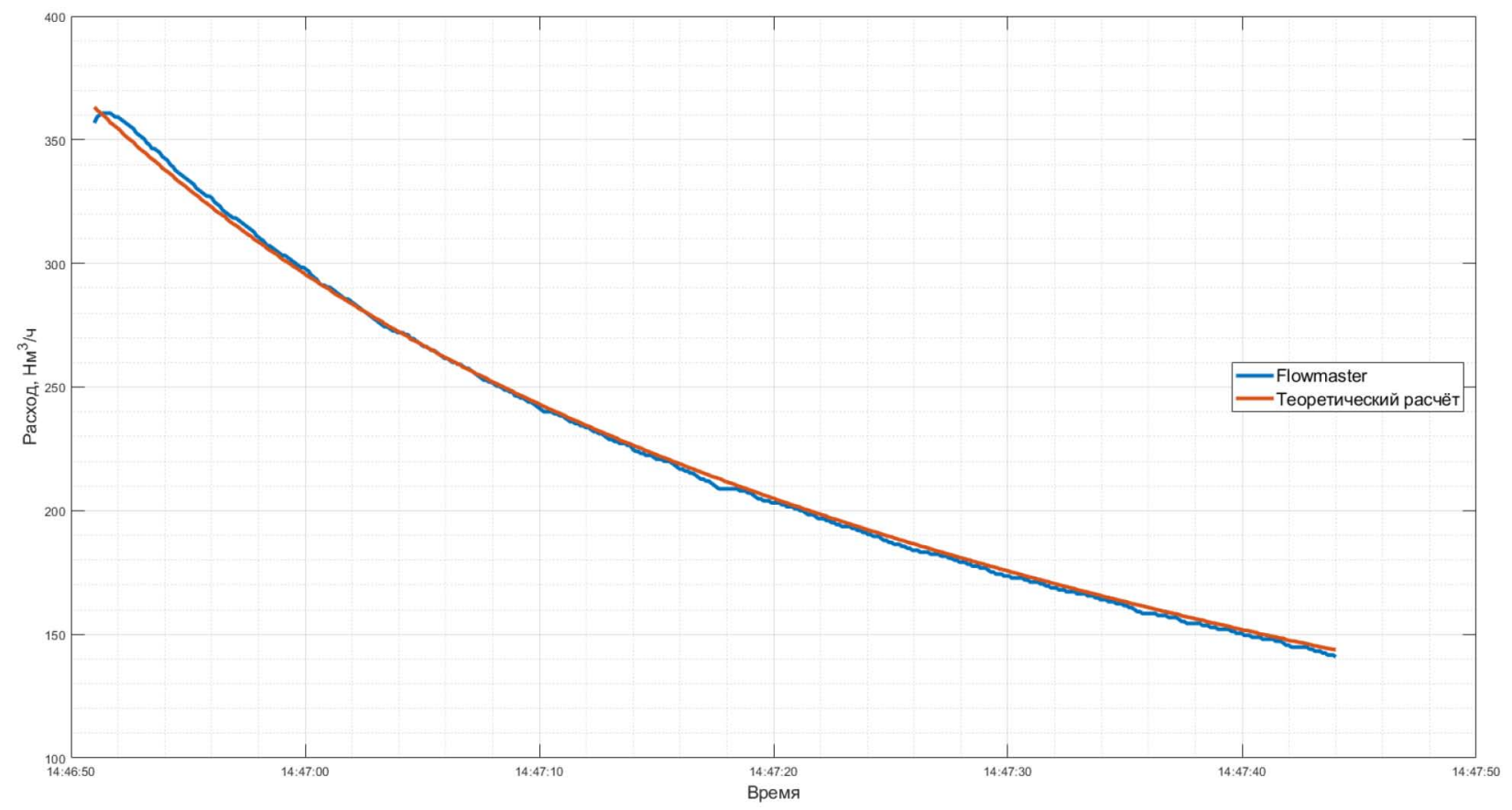

Figure 13-Flow meter readings for pressure relief through a throttle washer with a critical diameter of $3 \mathrm{~mm}$

\section{Conducting pilot works on the wells of the Orenburg oil and gas condensate field}

From January 2019, within 180 days, pilot works were carried out at the wells of the Orenburg oil and gas condensate field to test the "FlowMaster" for operation in a heterogeneous type of fluid (Figure 14). Field tests were carried out over a period of time, covering the winter, spring and summer, which allowed equipment testing in critical temperature conditions. Figures 15 and 16 show a comparison of gas-lift gas flow in normal and linear conditions. According to the results of the tests, it was shown that the flow meter based on a Venturi tube allows measuring the gas flow rate with the required accuracy and can operate in a heterogeneous medium (the presence of a liquid phase in a gas flow).

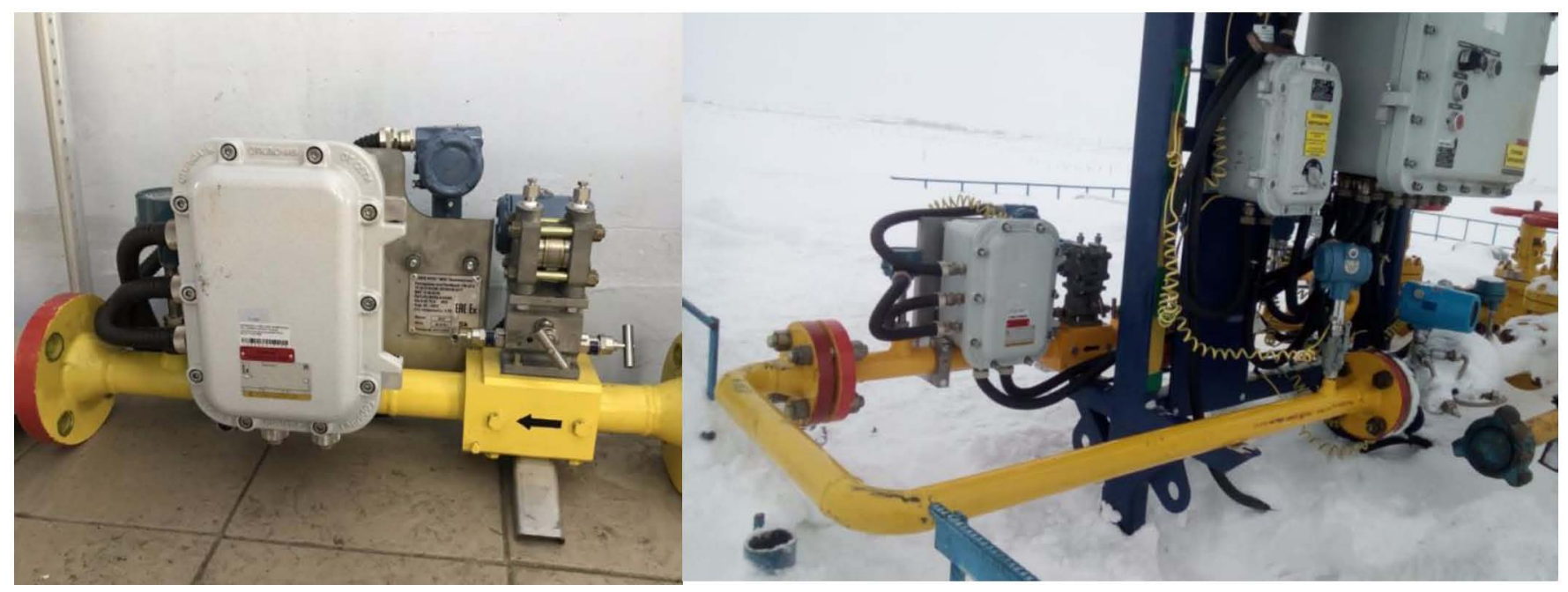

Figure 14-Conducting pilot work on the wells of the Orenburg oil and gas condensate field 


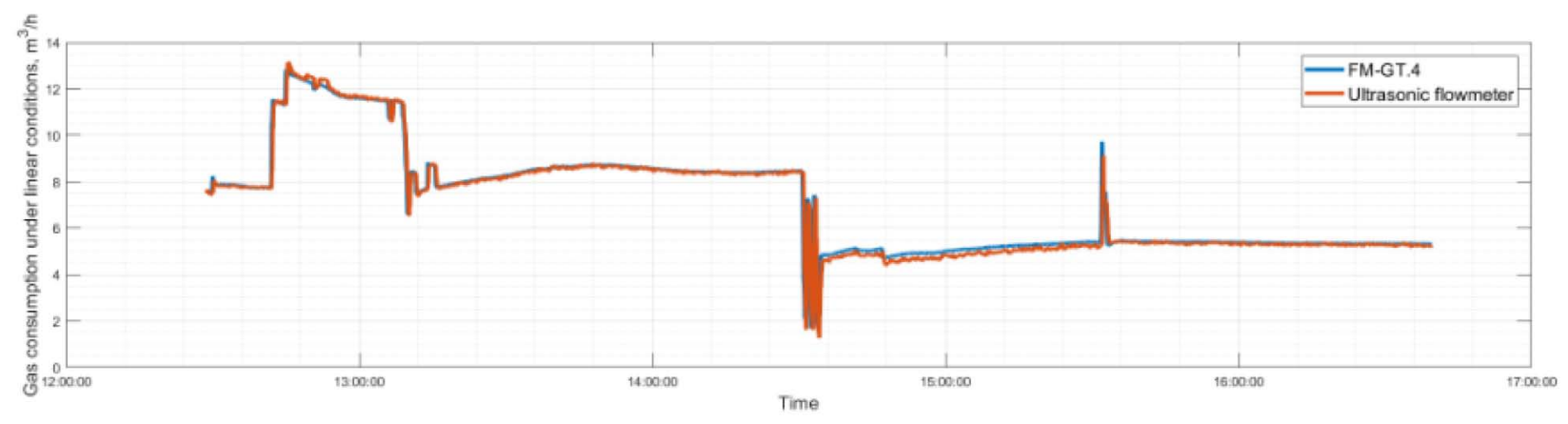

Figure 15-Comparison of measurements of gas-lift gas flow on an ultrasonic flow meter and a flow meter based on a Venturi tube in linear conditions

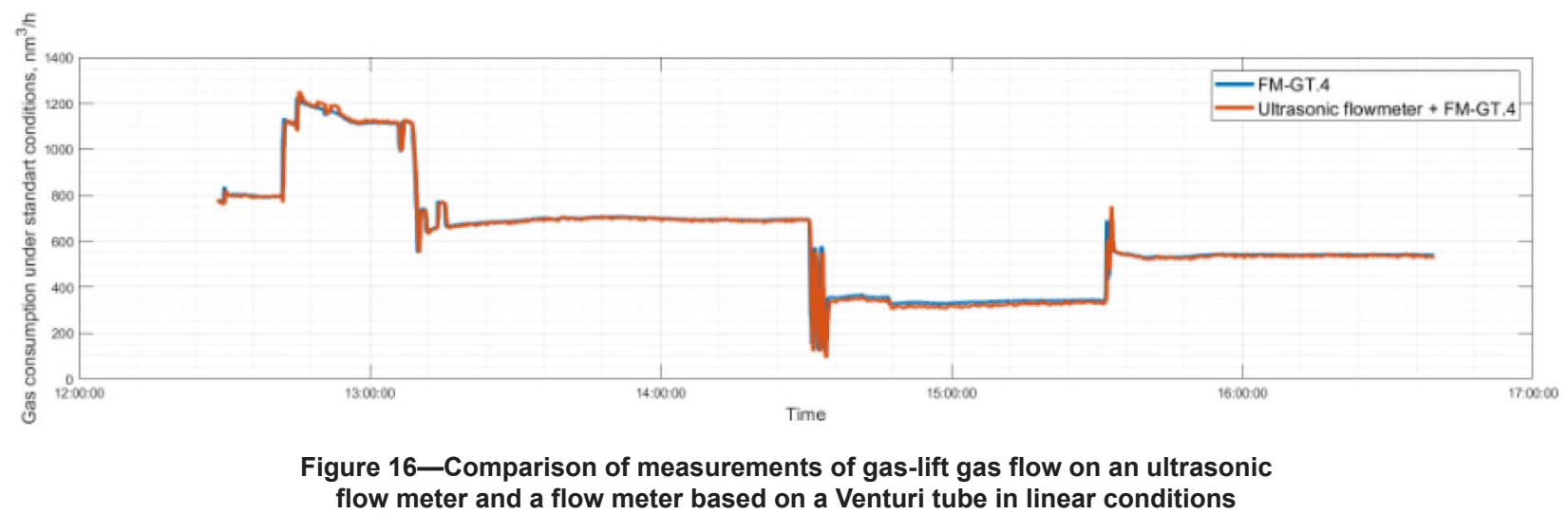

\section{Conclusion}

In the process of analyzing the results of using different types of gas-lift gas flowmeters under conditions of the Orenburg oil and gas condensate field, the following conclusions were reached:

- Due to the peculiarities of the channel geometry of the vortex flow meters, moisture accumulates behind them on sharply protruding elements and stagnant zones, which subsequently leads to accelerated precipitation of hydrates

- Ultrasonic flowmeters quite well show themselves in a homogeneous environment, but under conditions of unprepared gas, ultrasound scattering can occur on condensed phase wells in the Orenburg oil and gas condensate field, which creates difficult predictable oscillations

- The use of restriction devices such as diaphragms and nozzles lead to significant pressure losses and the formation of stagnant zones, which also leads to the deposit of gas hydrates

- The use of a flow meter based on a Venturi tube has shown stable operation in the presence of multiphase flows.

\section{References}

1. Galyautdinov I.M., Cherepovitsyn A.E. Complex approach to the selection of well-candidates for conducting geological and technical measures (by the example of the Eastern section of the Orenburg OGCC) // Oil. Gas. Innovations. - 2017. - № 7. - p. 23 - 33.

2. Trubavin S.N., Ulyanov V.V., Kibirev Ye.A., Rymarenko K.V., Bak V.S., Historin P.E., Nukhaev M.T. Results of the OPI for optimization of gas lift operation wells at the Orenburg OGKM / Exposure Oil Gas. - 2017. - № 5 (58), September. - with. 36-39. 
3. Shushakov A.A, N.M. Pavlechko, E.A. Kibirev, Ya.A. Burtsev, R.A. Khabibullin, V.V. Kuznetsov / "Optimizing gas lift wells in conditions of Gazpromneft Orenburg JSC with a new calculation module" // Oil-industry journal \# 12, 2015 p. 68-70.

4. Aksenov M., D. Lachugin, M. Nukhaev, K. Rymarenko, V. Telkov, L. Gaidukov, K. Vologodskiy, D. Khramov: SPE SPE-165427-MS "Specifics and Challenges of Heavy oil Production in Northen Siberia Illustration Based on Biggest Heavy Oil Project in Russia" // 2013

5. Ulyanov Vladimir, Kuchurin Alexey, Kibirev Evgeny, Gryzunov Andrey, Bak Viktor, Rymarenko Konstantin, Nukhaev Marat, Dadakin Nikita / "Implementation of the Intellectual Gas Control System for Gas Lift Optimization at Orenburgskoe Oilfield" // SPE paper 191533 